\title{
La inteligencia emocional como predictora del rendimiento académico: el contexto pluricultural de Ceuta
}

\section{The emotional intelligence as predictor of academic achievement: the pluricultural context of Ceuta}

\author{
Federico Pulido Acosta ${ }^{1}$, Francisco Herrera Clavero ${ }^{2}$ \\ ${ }^{1}$ Departamento de Psicología Evolutiva y de la Educación, Universidad de Granada, España (fe7de@ correo.ugr.es) \\ ${ }^{2}$ Departamento Psicología Evolutiva y de la Educación, Universidad de Granada, España (fherrera@ugr.es)
}

Recibido el 6 de noviembre de 2015; revisado el 20 de noviembre de 2015; aceptado el 21 de noviembre de 2015; publicado el 14 de diciembre de 2015

\begin{abstract}
RESUMEN:
Este trabajo tiene como objetivo fundamental conocer los predictores dela inteligencia emocional (IE) y el rendimiento académico (RA) del alumnado de la ciudad de Ceuta, para lo que se contó con 1186 participantes, de 9 centros educativos distintos, que reflejan la pluriculturalidad de la ciudad; de los cuales, el $57.8 \%$ son mujeres y el $42.2 \%$ varones, y el $58.9 \%$ de cultura/religión musulmana y el $41.1 \%$ cristianos. Como instrumentos de evaluación se emplearon una adaptación propia del MSCEIT (Test de Inteligencia Emocional de Mayer, Salovey y Caruso, 2009) y las calificaciones de los alumnos. Los resultados obtenidos reflejan niveles medios de RA y medio-altos en IE, actuando como predictores del primero las variables sociodemográficas de edad, género, cultura/religión y estatus socioeconómico-cultural. Los predictores de la IE son la etapa y el estatus. Por otra parte, muestran que existe relación entre IE y RA, actuando cada una como principal predictor de la otra.
\end{abstract}

PALABRAS CLAVE: EMOCIÓN, INTELIGENCIA EMOCIONAL, DESARROLLO EMOCIONAL, RENDIMIENTO ACADÉMICO, CONTEXTO PLURICULTURAL.

\section{ABSTRACT:}

The objective of this paper is to reflect the predictors of Emotional Intelligence (EI) and Academic Achievement (AA) and the influence over each other, of students in the city of Ceuta. We focused on 1186 participants from 9 different educational institutions; $42.2 \%$ are boys and $57.8 \%$ girls; $58.9 \%$ are Muslims and $41.1 \%$ Christians. The techniques used in this survey are a similar adaptation of MSCEIT (Mayer et al., 2009) to each one of the groups as well as the student's grades. The results show medium levels on AA and medium-high levels on EI. The predictors of AA are the sociodemographic factors of age, gender, customs/religion and socioeconomic and cultural. The predictors of EI are age and status. We found a relationship between EI and AA. Each one is the most important predictor of the other.

\section{KEYWORDS: EMOTION, EMOTIONAL INTELLIGENCE, EMOTIONAL DEVELOPMENT, ACADEMIC ACHIEVEMENT, PLURICULTURAL CONTEXT.}

\section{INTRODUCCIÓN}

La cambiante actualidad obliga a los sistemas educativos a retos cada vez más profundos y audaces, para adaptarse a nuevas formas culturales, sociales y políticas de manera vertiginosa, con el fin último de que el alumnado pueda aprender y desarrollar nuevas habilidades y capacidades que le permita afrontar el futuro con relativa esperanza (Codero y Manchón, 2014). En ese sentido, cada vez son más las voces que cuestionan la 
exclusividad de la inteligencia racional, como factor más influyente para alcanzar el éxito académico, entendiendo que existen otros que también habría que tener en cuenta, como por ejemplo las inteligencias emocional (Filella, Pérez, Agulló y Oriol, 2014) y social.

Tradicionalmente, se tendía a vivir como si todo dependiera de nuestro exterior, de elementos que están fuera de nosotros mismos, llegando incluso a rechazar la importancia de ese mundo interior, al margen de la inteligencia racional. Dentro de esos elementos internos, los estados emocionales comienzan a adquirir una importancia cada vez mayor, van haciéndose un hueco en las más diversas áreas de la vida. Las diferencias son claras entre IE e inteligencia racional, en línea con en el estudio realizado por Jones-Schenk y Harper (2014), donde se indica la independencia de ambas y se desligan claramente. Resulta difícil precisar qué tanto por ciento del éxito personal, académico y profesional corresponde al campo de las emociones. En cualquier caso, una importante parte del mismo se puede relacionar con esas estrategias emocionales empleadas por aquellos que integran este componente en sus vidas.

Por otro lado, aparece el concepto de IE, entendida como la habilidad para manejar el propio repertorio emocional, pasando el éxito del ámbito cognitivo al ámbito emocional. Resulta evidente que la manera de entenderla se centra en esta en cuanto a un conjunto de habilidades (no de forma percibida). Entre las habilidades específicas que integran la IE (Pulido y Herrera, 2015) quedan englobadas las habilidades de conocimiento de sí mismos (entendido como la adecuada identificación de las emociones propias), autocontrol (manejo de las propias emociones) y motivación (relacionada con el impulso director para el desarrollo de una tarea), empatía (capacidad de identificar las emociones en los demás) y habilidades sociales (habilidades que permiten la adecuada interacción con los demás).

Otro de los aspectos más relevantes en relación con la IE es la gran controversia existente con respecto a la influencia del género en esta. Desde este punto de vista, se parte de diferentes trabajos que plantean que las mujeres manifiestan niveles superiores (Billings, Downey, Lomas, Lloyd y Stough, 2014; Pulido y Herrera, 2015), lo que no hace otra cosa que alimentar el estereotipo de que las mujeres son más "emocionales" que los varones. Sin embargo, en otros estudios se demuestra que las diferencias en función del género están mediatizadas por factores diferentes. Entre estos factores se sugiere la variable edad (Billings et al., 2014) o la identidad de género (Gartzia, Aritzeta, Balluerka y Barberá, 2012).

Se pueden distinguir dos tipos de instrumentos para evaluar la IE: el primero se refiere a cómo las emociones y el pensamiento interactúan, como el de Mayer et al. (2009). Los modelos percibidos (autopercepción de la IE), utilizan inventarios de autoinforme.

Como concepto final, se define el RA, que se refiere al nivel de conocimientos y destrezas escolares exhibidos por los estudiantes. En el presente trabajo, se han empleado las calificaciones escolares como indicativo de este, considerando a Gómez Castro (1986, p. 269), ya que "al comparar los resultados de las calificaciones objetivas a través de una prueba estandarizada y las que otorgan los profesores, indica cierta asociación entre ellas. Todas las correlaciones obtenidas entre las distintas subpruebas y las calificaciones son estadísticamente significativas ( $p>.01)$ ". La cultura y el estatus socio económico y cultural ejercen una importante influencia en el RA (Pulido y Herrera, 2015).

Se contempla una relación directa entre IE y rendimiento, apareciendo relaciones evidentes entre ambos, en los trabajos de Billings et al. (2014), Brouzos, Misailidi y Hadjimattheou (2014) y Pulido y Herrera (2015). Esta idea pretende ser una de las bases del estudio que se pretende realizar.

Considerando todo lo mencionado, se presenta el estudio llevado a cabo con una muestra de alumnos escolarizados en centros de educación infantil y primaria, en institutos de enseñanza secundaria y en la Facultad de Educación y Humanidades en la ciudad de Ceuta, donde conviven principalmente personas de cultura cristiana y musulmana. Como característica específica, la población árabe musulmana presenta una procedencia marroquí con un altísimo nivel de analfabetismo y una elevada natalidad, así como un estatus socioeconómico y cultural bajo, serios problemas de enculturación y bilingüismo (integración por la comunicación) y con una presencia muy marcada de su religión, el Islam, en sus vidas. Esto nos lleva a establecer una clara relación entre la cultura y la religión.

Los objetivos del trabajo han sido conocer y analizar cuáles son los predictores de IE y RA en este alumnado, en función de variables como el género, la edad, la cultura y el estatus socioeconómico y cultural, así como conocer y analizar qué relación existe entre las mencionadas variables de estudio. En base a la revisión bibliográfica realizada se esperan resultados superiores en los niveles de IE en función del género y la edad (etapa). En RA también se esperan diferencias en 
función de la cultura y el estatus socioeconómico y cultural. Se espera obtener niveles superiores en rendimiento entre los sujetos con niveles más elevados de IE.

\section{MATERIAL Y MÉTODO}

La muestra estuvo integrada por 1186 participantes de 9 centros educativos (4 de E. Primaria, 4 de E. Secundaria y la Facultad de Educación, Economía y Tecnología de Ceuta). El $34 \%$ de la muestra correspondía a centros de educación infantil y primaria (alumnado infantil), el $47 \%$ a institutos de enseñanza secundaria (alumnado adolescente) y el restante 19\% al ámbito universitario (alumnado adulto). Considerando la cultura (que se corresponde con la religión), el grupo mayoritario fue el de participantes pertenecientes a la cultura/religión musulmana, el 58.9\% de la muestra, y el $41.1 \%$ de cultura-religión cristiana, lo que representa las culturas mayoritarias de la ciudad, no teniendo en cuenta miembros de otras culturas por su reducido número. En función de la variable género, de porcentajes parecidos, el $42.2 \%$ eran varones y el $57.8 \%$ mujeres. Por estatus socioeconómico y cultural, un $15.2 \%$ correspondía a un estatus bajo, un $31 \%$ medio-bajo, un $43.2 \%$ medio y el restante $10.6 \%$ con un estatus alto. Se seleccionó un curso en cada uno de los centros de Primaria (de primero a sexto), Secundaria (de primero de ESO a segundo de bachillerato) y la Facultad (desde primero hasta cuarto curso de grado, por cada una de las carreras), aplicándose ambos cuestionarios durante la primera hora de clase. Antes de aplicar las diferentes pruebas se explicó cómo debían contestar y se aclararon las dudas reflejadas por cada uno de los participantes. El error muestral fue del $3 \%$.

Como instrumentos para evaluar la IE se empleó una adaptación del MSCEIT (Test de Inteligencia Emocional de Mayer, Salovey y Caruso, 2009) a la población infantil, adolescente y adulta. Para llevar a cabo la adaptación se siguió la misma estructura del cuestionario original, respetando las diferentes secciones del mismo (exceptuando la $G$, que se suprimió por su excesiva dificultad para el alumnado de Primaria y Secundaria). En ellas aparecen apartados para la identificación de emociones en gestos faciales $y$ en diferentes fotografías (secciones A y $\mathrm{E}$ del cuestionario original), la comprobación de la utilidad de diferentes emociones en situaciones determinadas (sección B), la selección de la emoción que más se ajuste a cada caso (sección C), la respuesta a cuestiones emocionales acordes a determinadas historias (sección D y H) y la relación de diferentes emociones con acciones concretas (sección F); ajustando las diferentes secciones a la dificultad del alumnado. En todos los casos, los ítems se clasificaron en cinco factores: motivación, empatía, autocontrol emocional, conocimiento de uno mismo y autoconcepto. Explicando el $29.10 \%$ de la varianza total, para la muestra infantil; del $23.87 \%$, para la adolescente, y de $24.64 \%$, para los universitarios.

Finalmente, se emplearon las calificaciones medidas del RA. Dado que existía diversidad en cuanto a las materias evaluadas, se reagruparon conformando 6 categorías diferentes. En este sentido, los bloques empleados fueron los siguientes: la materia (área/asignatura) 1, compuesta por las asignaturas de Lengua Castellana (Primaria y Secundaria) y Psicología/Métodos Cuantitativos en el caso de los adultos. La materia 2 integra las asignaturas de Matemáticas (Primaria y Secundaria) y Sociología/Creación de Empresas en la Universidad. La materia 3 queda conformada por Conocimiento del Medio (Primaria), Ciencias Sociales (Secundaria) y Educación Artística/Contabilidad Financiera en la Facultad de Educación y Humanidades. Para la materia 4 se unen las asignaturas de Inglés, así como por Didáctica/Contabilidad de Costes (en la población Adulta). La materia 5 la forman Religión/Ciudadanía (Primaria y Secundaria) y Organización. Para la materia 6, dado que en Primaria solo se recogió la calificación de 5 asignaturas, esta última materia queda integrada por Ciencias Naturales (adolescentes) y Educación (adultos).

Tras solicitar y obtener las pertinentes autorizaciones, tanto en la Dirección Provincial de Educación, como en cada uno de los diferentes centros, se pasaron los cuestionarios. Se respetó escrupulosamente la confidencialidad. Siendo previamente informados, los alumnos, voluntariamente, contestaron los cuestionarios. Todos los cuestionarios fueron pasados por el investigador, en los diferentes centros educativos, en presencia del profesor de cada aula.

Una vez construida la base de datos, se comenzó por el análisis descriptivo, donde se destacaron los porcentajes alcanzados de manera general y en función de las variables sociodemográficas, a través de tablas de contingencia. Para comprobar los predictores se muestran los resultados de la regresión múltiple paso a paso o "stepwise" realizada, considerando como variable criterio la IE 
y el RA, así como cada una de las materias que forman parte del último. Como predictoras se han usado las sociodemográficas y la que no actuaba como variable criterio. El análisis completo ha permitido la comprobación del comportamiento de la muestra desde la perspectiva las variables sociodemográficas, así como la interacción entre las variables de estudio.

\section{RESULTADOS}

Con respecto a las puntuaciones totales de IE se puede observar que la opción mayoritaria es la que refleja bastante IE, alcanzada por el $63.9 \%$ de la muestra. La siguiente categoría, es la que evidencia poca IE, con el $18.5 \%$ de la muestra, continuando, la que refleja los niveles más altos de IE (mucha) con el $14 \%$ de la muestra total. El restante $3.6 \%$ de la muestra refleja muy poca IE. Se observan, por tanto un nivel general "medio-alto".

Los resultados resumidos en la tabla 1 muestran que la IE está bajo la influencia de las variables Rendimiento Total, Etapa y Estatus. El conjunto de ellas da cuenta del $25.3 \%$ de la varianza en IE ( $\mathrm{R} 2=0253)$. El mayor poder determinante lo ejerce la variable RA, que por sí solo explica el $37.9 \%$ de la varianza en IE $(\beta=0.379)$. Existe una relación directamente proporcional entre la IE y el RA, aumentando las puntuaciones en IE a medida que lo hacen las calificaciones. Lo mismo ocurre con la Etapa y el Estatus, registrándose niveles superiores a medida que aumenta la edad (Etapa) y el Estatus socioeconómico y cultural. El resto de variables quedaron rechazadas. Siguiendo la función que aparece a continuación, la regresión hace posible calcular la puntuación en Inteligencia Emocional, conocida la calificación total, la edad del sujeto y su Estatus.

$$
\begin{gathered}
\text { IE Total }=1.319+.145(\text { Rendimiento Total })+.243 \\
(\text { Etapa })+.076(\text { Estatus })
\end{gathered}
$$

Tabla 1. Regresión Inteligencia Emocional

$$
\mathrm{R}=.503 \mathrm{R} 2=.253 \mathrm{~F}=43.019 \mathrm{p}=. \neg 0 \neg 000
$$

\begin{tabular}{ccccc}
\hline Variables & $\mathrm{B}$ & $\beta$ & $\mathrm{t}$ & $\mathrm{p}$ \\
\hline $\begin{array}{c}\text { Constante } \\
\text { General }\end{array}$ & 1.319 & & 15.597 & .0000 \\
$\begin{array}{c}\text { Rendimiento } \\
\quad \text { Total }\end{array}$ & .145 & .379 & 14.122 & .0000 \\
$\quad \begin{array}{c}\text { Etapa } \\
\text { Evolutiva } \\
\text { Estatus }\end{array}$ & .243 & .255 & 9.849 & .000 \\
\hline
\end{tabular}

Por edad, las diferencias resultan estadísticamente significativas en IE Total $(\mathrm{p}=0.000)$, observándose un aumento progresivo en los niveles a medida que se asciende en edad. Así la etapa con niveles mayores es la edad adulta, seguida de la adolescencia y terminando por la etapa infantil. Los participantes pertenecientes al grupo de Cultura/Religión musulmana presentan peores resultados en IE (1.64 frente a 1.85 de media). Por otra parte, los Estatus más altos se asocian a un mayor manejo emocional, ya que se observa que el nivel en el que aparecen las puntuaciones superiores es en el medio (1.86), seguido del alto (1.67) que presenta puntuaciones menores. Después vendría el medio-bajo (1.63). Con los niveles menores, aparece el estatus bajo (1.54). El hecho de pertenecer a un determinado estatus influye en la IE Total $(\mathrm{p}=0.000)$.

En segundo lugar, para el RA, expresado por la nota media, el resultado más repetido $(34.6 \%)$ es el notable. La siguiente calificación, por porcentaje, es el "suspenso" (22.5\%). A continuación, muy cerca, aparece la calificación "bien" (19.9\%) y después el sobresaliente (con un 13.5\%). La calificación con porcentajes más bajos (9.5\%) es el aprobado.

Los resultados de la regresión múltiple (presentes en la tabla 2) indican que el RT está bajo la influencia de las variables IE, Cultura, Etapa, Estatus y Género. El conjunto de ellas da cuenta del $25.8 \%$ de la varianza en Rendimiento general $(\mathrm{R} 2=0.258)$. El mayor poder determinante lo tiene la Inteligencia Emocional, explicando el $38 \%$ de la varianza en Rendimiento Total $(\beta=0.380)$. El RA es directamente proporcional a la IE, de la misma manera que ocurre con el Estatus y el Género. A mayor dominio emocional y nivel socioeconómico y cultural, mayores son las calificaciones. Para el Género, las puntuaciones son superiores entre las chicas (esto explica que el valor sea positivo, ya que al Género masculino se le dio el valor 1 y al femenino el valor 2). Las variables Cultura/Religión y Etapa actúan de manera inversa, descendiendo las calificaciones entre los musulmanes (se asoció este grupo cultural con el valor numérico 2) y a medida que se asciende en el edad (Etapa). Se puede predecir el Rendimiento, considerando la función: 
Rendimiento Total $=4.360+.998$ (IE) -.778

(Cultura/Religión $)-.293($ Etapa $)+.225$ (Estatus $)+.200$

(Género)

Tabla 2. Regresión Rendimiento Total.

$\mathrm{R}=.508 \mathrm{R} 2=.258 \mathrm{~F}=78.150 \mathrm{p}=. \neg 0 \neg 000$

\begin{tabular}{ccccc}
\hline Variables & $\mathrm{B}$ & $\beta$ & $\mathrm{t}$ & $\mathrm{P}$ \\
\hline Constante & 4.360 & & 11.635 & .0000 \\
$\quad$ General & & & & \\
Inteligencia & .998 & .380 & 13.837 & .0000 \\
Emocional & & & & \\
Cultura/Religión & -.778 & -.218 & -7.390 & .000 \\
Etapa & -.293 & -.117 & -4.235 & .000 \\
Estatus & .225 & .108 & 3.707 & .000 \\
Género & .200 & .056 & 2.149 & .032 \\
\hline
\end{tabular}

La regresión arroja los datos (que se muestran en la tabla 3) que permiten ver como el Rendimiento en la Materia 1 está también bajo la influencia de la IE y de las variables Cultura/Religión, Estatus, Género y Etapa. Entre todas dan cuenta del $18.9 \%$ de la varianza esta Materia $(\mathrm{R} 2=0.189)$. La variable más determinante resulta, de nuevo, la IE, que por sí sola explica el $27.5 \%$ de la varianza $(\beta=0.275)$. Como ocurre en casos ya comentados, la relación es directamente proporcional entre el RA y la IE, de la misma manera que ocurre con el Género y el Estatus (calificaciones más altas entre los niveles altos y las chicas). De nuevo, la Cultura y la Etapa adquieren valores negativos, disminuyendo el Rendimiento a medida que se asciende en edad. Los musulmanes obtienen calificaciones más bajas. Todo ello se puede observar en la siguiente función, en la que aparecen los predictores del Rendimiento en la Materia 1.

Rendimiento Materia 1= $4.235+.866$ (IE) - .934 (Cultura/Religión $)+.276$ (Estatus $)+.361$ (Género $)-$ .256 (Etapa)

Tabla 3. Regresión Rendimiento Materia 1

$$
\mathrm{R}=.435 \mathrm{R} 2=.189 \mathrm{~F}=52.548 \mathrm{p}=. \neg 0 \neg 000
$$

\begin{tabular}{ccccc}
\hline Variables & $\mathrm{B}$ & $\beta$ & $\mathrm{t}$ & $\mathrm{P}$ \\
\hline Constante & 4.235 & & 9.011 & .000 \\
$\quad$ General & & & & \\
Inteligencia & .866 & .275 & 9.576 & .000 \\
Emocional & & & & \\
Cultura/Religión & -.934 & -.218 & -7.070 & .000 \\
Estatus & .276 & .110 & 3.626 & .000 \\
Género & .361 & .084 & 3.098 & .002 \\
Etapa & -.256 & -.085 & -2.943 & .003 \\
\hline
\end{tabular}

En la tabla 4 se reflejan los resultados que permiten demostrar que el Rendimiento en la Materia 2 está bajo la influencia de las variables IE, Cultura/Religión y Estatus, siendo la primera y la última directamente proporcionales. El conjunto de ellas dan cuenta del $19.5 \%$ de la varianza en esta disciplina $(\mathrm{R} 2=0.195)$. El mayor poder determinante lo ejerce, de nuevo, la IE que explica el $29.4 \%$ de la varianza total $(\beta=0.294)$. A mayor manejo emocional y Estatus, más altas son las calificaciones. Para la Cultura, los valores tomados vuelven a ser negativos, lo que indica que son los cristianos los que obtienen un rendimiento superior. Las variables Género y Etapa quedan rechazadas en esta ocasión. La función que se observa a continuación permite averiguar la puntuación en la Materia 2, conocidas las demás variables.

Rendimiento Materia 2=3.952 + .976 (IE) -.899 (Cultura/religión) +.294 (Estatus)

Tabla 4. Regresión Rendimiento Materia 2 $\mathrm{R}=.442 \mathrm{R} 2=.195 \mathrm{~F}=91.061 \mathrm{p}=. \neg 0 \neg 000$

\begin{tabular}{ccccc}
\hline Variables & $\mathrm{B}$ & $\beta$ & $\mathrm{t}$ & $\mathrm{P}$ \\
\hline Constante & 3.952 & & 8.920 & .000 \\
General & & & & \\
Inteligencia & .976 & .294 & 10.621 & .000 \\
Emocional & & & & \\
Cultura/Religión & -.889 & -.197 & -6.536 & .000 \\
Estatus & .294 & .111 & 3.676 & .000 \\
\hline
\end{tabular}

Para el Rendimiento en la Materia 3, los resultados de la regresión (tabla 5) permiten predecir sus puntuaciones si se conocen las variables IE, Cultura/Religión y Estatus. Todas ellas suman el $15.5 \%$ de la varianza en este factor $(\mathrm{R} 2=0.155)$. El mayor poder determinante lo ejerce la IE que explica el $29.3 \%$ de la varianza $(\beta=0.293)$. Para todas las variables, excepto la relacionada con la Cultura/Religión, se describe una relación directamente proporcional, explicada en anteriores casos. En el caso de la Cultura, los musulmanes obtienen resultados más bajos (de ahí su valor negativo). Con la función que se puede describir con estos datos es posible calcular cualquiera de las variables conocidas las demás.

$$
\begin{gathered}
\text { Rendimiento Materia } 3=3.872+.934 \text { (IE)- } .516 \\
\text { (Cultura/Religión) }+.295 \text { (Estatus) }
\end{gathered}
$$

Tabla 5. Regresión Rendimiento Materia 3.

$$
\mathrm{R}=.394 \mathrm{R} 2=.155 \mathrm{~F}=69.030 \mathrm{p}=.000
$$

\begin{tabular}{ccccc}
\hline Variables & $\mathrm{B}$ & $\beta$ & $\mathrm{T}$ & $\mathrm{P}$ \\
\hline Constante & 3.872 & & 8.893 & .000 \\
$\quad$ General & & & & \\
Inteligencia & .934 & .293 & 10.343 & .0000 \\
Emocional & & & & \\
Cultura/Religión & -.516 & -.119 & -3.858 & .000 \\
$\quad$ Estatus & .295 & .116 & 3.756 & .000 \\
\hline
\end{tabular}


En el rendimiento en la Materia 4, los cálculos de regresión reflejan que la IE y la Cultura/Religión son las variables que ejercen influencia. Todas las variables predictoras representan el $14.5 \%$ de la varianza en Rendimiento en esta Materia $(\mathrm{R} 2=0.145)$. El mayor poder determinante vuelve a tenerlo la IE, que por sí sola explica el $14.5 \%$ de la varianza $(\beta=0.145)$. Existe una relación directamente proporcional entre el RA y la IE, lo contrario que ocurre con la Cultura/Religión, donde los cristianos obtienen mejores resultados en la materia. Los datos que han permitido realizar esta descripción aparecen resumidos en la tabla 6.

$$
\begin{gathered}
\text { Rendimiento Materia 4= } 4.197+1.027 \text { (IE) - .652 } \\
\text { (Cultura/Religión) }
\end{gathered}
$$

Tabla 6. Regresión Rendimiento Materia 4

$$
\mathrm{R}=0.381 \mathrm{R} 2=0.145 \mathrm{~F}=95.882 \mathrm{p}=0 . \neg 0 \neg 000
$$

\begin{tabular}{ccccc}
\hline Variables & $\mathrm{B}$ & $\beta$ & $\mathrm{t}$ & $\mathrm{P}$ \\
\hline Constante & 4.197 & & 11.658 & .0000 \\
$\begin{array}{c}\text { General } \\
\text { Inteligencia }\end{array}$ & 1.027 & .321 & 11.380 & .0000 \\
$\begin{array}{c}\text { Emocional } \\
\text { Cultura/Religión }\end{array}$ & -.652 & -.150 & -5.325 & .000 \\
\hline
\end{tabular}

Para concluir con las Materias, se muestran los datos reflejados en la tabla 7 , los cuales permiten calcular el Rendimiento en la disciplina 5. En esta ocasión, la IE, la Etapa, el Estatus y el Género son las variables que ejercen influencia, dando cuenta del $21.9 \%$ de la varianza total $(\mathrm{R} 2=0.219)$. La IE explica el $33.8 \%$ de la varianza total en esta Materia $(\beta=0.338)$. El resto de variables quedan rechazadas. En este caso los valores positivos de IE, Estatus y Género indican que son más altas las calificaciones entre los que presentan mayores niveles de IE, los que ocupan un Estatus más alto y las chicas. Para la Etapa, los valores negativos indican que son los más jóvenes los que obtienen puntuaciones más elevadas. La función que explica todo lo comentado aparece a continuación.

Rendimiento Materia 5= 5.369+1.004 (IE) -.790

$($ Etapa $)+.282$ (Estatus) +.251 (Género)

Tabla 7. Regresión Rendimiento Materia 5.

\begin{tabular}{ccccc}
\multicolumn{5}{c}{$\mathrm{R}=0.467 \mathrm{R} 2=0.219 \mathrm{~F}=62.303 \mathrm{p}=0 . \neg 0 \neg 000$} \\
\hline Variables & $\mathrm{B}$ & $\beta$ & $\mathrm{t}$ & $\mathrm{P}$ \\
\hline Constante & 5.369 & & 12.283 & .0000 \\
$\quad$ General & & & & \\
Inteligencia & 1.004 & .338 & 11.946 & .0000 \\
Emocional & & & & \\
$\quad$ Etapa & -.790 & -.196 & -6.426 & .000 \\
Estatus & .282 & .120 & 3.980 & .000 \\
Género & .251 & .062 & 2.314 & .021 \\
\hline
\end{tabular}

Entre los predictores de la IE se observa que las calificaciones son claramente superiores entre los cristianos, ya que superan a los musulmanes en Rendimiento medio general. Los resultados de la estadística inferencial demuestran que la cultura influye en la Media de Rendimiento Total $(\mathrm{p}=0.000)$, el Rendimiento en la Materia 1 $(\mathrm{p}=0.000)$, el Rendimiento en la Materia 2 $(\mathrm{p}=0.000)$, el Rendimiento en la Materia 3 $(\mathrm{p}=0.000)$, el Rendimiento en la Materia $4(\mathrm{p}=0.000)$ y el Rendimiento en la Materia $5(\mathrm{p}=0.000)$. En todos los casos, el grupo de cristianos vuelve a obtener mejores resultados que los musulmanes.

Considerando la Etapa evolutiva, se puede observar que las diferencias resultan estadísticamente significativas tanto en la Media de Rendimiento Total ( $\mathrm{p}=0.000)$, como en el Rendimiento en la Materia $1 \quad(p=0.000)$, el Rendimiento en la Materia $2 \quad(p=0.000)$, el Rendimiento en la Materia $3 \quad(p=0.000)$, el Rendimiento en la Materia $4(\mathrm{p}=0.000)$ y el Rendimiento en la Materia $5(\mathrm{p}=0.000)$.

Otro importante predictor del Rendimiento (y de la mayoría de Materias) es el Estatus. En Rendimiento Global, las más altas calificaciones se dan en los sujetos de Estatus alto (7.27), algo que refuerza la prueba ANOVA: Las diferencias son estadísticamente significativas en Media de Rendimiento Total ( $\mathrm{p}=0.000$ ), el Rendimiento en la Materia $1(\mathrm{p}=0.000)$, en la Materia $2(\mathrm{p}=0.000)$, en la Materia $3(\mathrm{p}=0.000)$, en la Materia $4(\mathrm{p}=0.000)$ y en la Materia $5(\mathrm{p}=0.000)$. Se comprueba que, a menor estatus, menores son también las calificaciones.

El Género actúa como predictor del Rendimiento Global, así como en dos materias. De esta manera, teniendo en cuenta la variable género, aparecen diferencias significativas en la Media de Rendimiento Total ( $\mathrm{p}=0.003)$, el Rendimiento en la Materia $1(\mathrm{p}=0.000)$, la Materia $2(\mathrm{p}=0.019)$, la Materia $3(\mathrm{p}=0.009)$, la Materia $4(\mathrm{p}=0.006)$ y la Materia 5 ( $\mathrm{p}=0.015)$. En todos los casos en los que las diferencias fueron significativas, las chicas obtuvieron resultados superiores.

Sin embargo, el predictor más importante en el Rendimiento (incluyendo cada una de las materias) es la Inteligencia Emocional. A medida que aumenta el nivel de IE aparecen calificaciones más altas. El grupo que presenta niveles más altos de IE presenta también mejores resultados (media de 7.80), encontrándose los porcentajes más altos en sobresalientes (44.4\%) y siendo el que tiene el menor porcentaje de suspensos (4.4\%). Después 
aparece el grupo que refleja bastante IE (6.43), con los segundos porcentajes más altos en sobresalientes $(9.8 \%)$ y primeros en notables $(40.9 \%)$. Aparecen similitudes entre el grupo que evidencia muy poca (5.29) y poca (5.15) IE, en los que aparecen los segundos y los mayores porcentajes en cuanto a suspensos ( $46.2 \%$ y $51.9 \%$ respectivamente). Esto queda más claro si se consideran las medias de cada materia, donde los que más IE presentan también tienen los resultados más altos (Materia 1 -7.49-, Materia 2 -7.73-, Materia 3 -7.94-, Materia 4 -7.62-, Materia 5 -8.23- y Materia 6 -7.97-). En el apartado inferencial (tabla 3 que aparece en la siguiente página), se constata la relación entre la Inteligencia Emocional y el Rendimiento, dado que se encontraron diferencias significativas la Media de Rendimiento Total ( $\mathrm{p}=0.000$ ), el Rendimiento en la Materia $1(\mathrm{p}=0.000)$, el Rendimiento en la Materia 2 $(\mathrm{p}=0.000)$, el Rendimiento en la Materia 3 $(\mathrm{p}=0.000)$, el Rendimiento en la Materia 4 $(\mathrm{p}=0.000)$, el Rendimiento en la Materia $5(\mathrm{p}=0.000)$ y el Rendimiento en la Materia $6(\mathrm{p}=0.000)$. En todos los casos los sujetos que tenían mayor nivel de Inteligencia Emocional.

\section{DISCUSIÓN/CONCLUSIONES}

La población refleja niveles medio-altos de IE. Entre los predictores de esta aparecen el Rendimiento, la edad (etapa evolutiva a la que el sujeto pertenece) y el Estatus (relacionado con la Cultura). Considerando la Etapa, los mayores alcanzan mejores resultados. Parece claro que el proceso de desarrollo emocional incide aumentando las habilidades emocionales, algo ya mencionado por Billings et al. (2014), Brouzos et al. (2014) y Pulido y Herrera (2015), evidenciándose que la maduración contribuye a la mejora de las diferentes capacidades que integran la IE. Por otra parte, los estatus más altos se asocian a un mayor manejo emocional. Relacionado con este factor, íntimamente dependientes aparecen diferencias por el grupo cultural (a pesar de que este factor no actúa como predictor), algo comentado en otros estudios (Billings et al., 2014 y Pulido y Herrera, 2015). En este sentido, se comentan las dificultades para la integración socio educativa (Oropesa, Moreno, Pérez y Muñoz-Tinoco, 2014) y las diferencias de Estatus socioeconómico y cultural (Hernández, Rodríguez y Moral, 2011) como importantes justificantes de estas diferencias significativas, como hallaron Cervini, Dari y Quiroz (2014) y Pulido y Herrera (2015). Esta diversidad encontrada en los núcleos familiares, con estilos de crianza distintos, junto con las importantes diferencias en la lengua materna (Pulido y Herrera, 2015; Siqués y Vila, 2014) entre ambas etnias, contribuyen a la aparición de problemas de integración socioeducativa del alumnado no autóctono.

En el Rendimiento, se dan niveles medios (bien de media). La materia en la que aparecen mejores resultados es en la 5 (en la que la nota media es notable). La materia 1 es en la que se registran los resultados más bajos. Entre los predictores aparecen la IE, la Cultura, la Etapa, el Estatus y el Género. La Cultura y el Estatus socio económico y cultural ejercen una importante influencia en las puntuaciones totales y en casi todas las materias, obteniendo los cristianos y los niveles altos resultados superiores. Para la Cultura, resultan importantes las diferencias en cuanto a la lengua materna entre musulmanes y cristianos para justificar estas diferencias. Esta explicación cobra más importancia si se considera el, ya mencionado, trabajo de Pulido y Herrera (2015), con alumnado perteneciente al mismo contexto. El contexto "autóctono" se mostraría como un impulso para el desarrollo socioafectivo. Ante esta situación, la interculturalidad se antoja necesaria aunque no suficiente para la progresiva eliminación de estas diferencias, convirtiendo ese contexto en "autóctono" para todos los grupos culturales. Así, la formación permanente del profesorado es la única vía para lograr armonizar la formación que reciben los docentes con la que deseamos que ofrezcan a sus alumnos en la sociedad intercultural que estamos construyendo (Goenechea, 2008). Por otra parte, el estatus también se ha de tener en cuenta, pues según el estudio de Alonso y Román, (2014) el nivel económico y sociocultural correlaciona positivamente con el estilo educativo familiar, y este, a su vez, con el nivel de autoestima mayor que repercute en diferentes dimensiones de desarrollo: ajuste emocional, éxito social y académico. La edad (Etapa) influye, disminuyendo la calificación conforme aumenta la primera. El Género también actúa como predictor, aunque solo en los totales y algunas materias, presentando las mujeres mejores resultados que los varones.

Un apartado especial merece la relación entre Inteligencia Emocional y Rendimiento Académico, ya que funcionan como principal predictor de la otra, de manera recíproca. Por eso, se concluye con la idea de que a mayor nivel de IE mayor Rendimiento Académico, lo que viene a confirmar los encontrados por diferentes autores como es el caso de Billings et al. (2014), Brouzos et al. (2014) y Codier y Odell (2014). La relación directamente 
proporcional entre la IE y el Rendimiento muestra claramente que las habilidades emocionales guardan una relevancia evidente con el logro académico y no solo para el desarrollo de los sujetos. Considerar y potenciar las competencias emocionales se antoja una medida necesaria para mejorar el proceso de aprendizaje del alumnado, situación que tanto se demanda a nuestro sistema educativo (De Haro y Castejón, 2014; Di Giusto, Martín, Arnaiz y Guerra, 2014). Esto es algo más evidente aun durante las primeras etapas educativas. Así nos situamos ante una importante alternativa a la inteligencia racional en la búsqueda de mejoras de los resultados académicos.

Por todo ello, considerar y potenciar las competencias emocionales se antoja una medida necesaria para mejorar el proceso de aprendizaje, lo que debería tener muy en cuenta nuestro Sistema Educativo. Trabajar las competencias emocionales se convierte en una alternativa de apoyo ante tal situación, en cualquiera de los niveles, referido al aprendizaje centrado en el alumno como elemento más importante del entorno educativo (Castevich, Dzib, Hogan, Sanders, Slovec y Yelizarov, 2015).

\section{REFERENCIAS}

Alonso, J., y Román, J. M. (2014). Nivel sociocultural, prácticas educativas familiares y autoestima de los hijos en edades tempranas. Revista de Investigación Educativa, 32(1), 187-202.

Billings, C., Downey, L. A., Lomas, J. E., Lloyd, J., y Stough, C. (2014). Emotional Intelligence and scholastic achievement in pre-adolescent children. Personality and Individual Differences, 65, 14-18.

Brouzos, A., Misalidi, P., y Hadjimatheou, A. (2014). Associations Between Emotional Intelligence, SocioEmotional Adjustment, and Academic Achievement in Childhood: The Influence of Age. Canadian Journal of School Psychology, 29(2), 83-99.

Castevich, J., Dzib, A., Hogan, D. L., Sanders, L., Slovec, K., y Yelizarov, D. (2015). Desarrollo del entorno personal de aprendizaje para tutoría e investigación en niveles educativos superiores. Innoeduca, 1(1), 10-16.

Cervini, R., Dari, N., y Quiroz, S. (2014). Estructura familiar y Rendimiento Académico en países de América Latina. Los datos del Segundo Estudio Regional Comparativo y Explicativo. Revista Mexicana de Investigación Educativa, 19(61), 569-597.

Codero, J. M., y Manchón, C. (2014). Factores explicativos del rendimiento en educación primaria: un análisis a partir de TIMSS 2011. Estudios Sobre Educación, 27, 9-35

Codier, E., y Odell, E. (2014). Measured emotional intelligence ability and grade point average in nursing students. Nurse Education Today, 34, 608-612.

De Haro, J. M., y Castejón, J. L. (2014). Perceived emotional intelligence, general intelligence and earlyprofessionalsuccess: predictive and incremental validity. Anales de Psicología, 30(2), 490-498
Di Giusto, C., Martín M. E., Arnaiz, A., y Guerra, P. (2014). Competencias personales y sociales en adolescentes. Revista Iberoamericana de Educación, 66, 89-104. Recuperado de http://www.rieoei.org/rie66a06.pdf.

Filella, G., Pérez, N., Agulló, M. J., y Oriol, X. (2014). Resultados de la aplicación de un programa de educación emocional en Educación Primaria. Estudios Sobre Educación, 26, 125-147.

Gartzia, L., Aritzeta, A., Balluerka, N., y Barberá, E. (2012). Inteligencia emocional y género: más allá de las diferencias sexuales. Anales de psicología, 28(2), 567575.

Goenechea, C. (2008). ¿Es la formación del profesorado la clave de la educación intercultural? Revista Española de Pedagogía, 239, 119-136.

Gómez-Castro, J. L. (1986). Rendimiento escolar y valores interpersonales: Análisis de resultados en EGB con el cuestionario SIV de Leonardo V. Gordon. Bordón, 262, 257-275.

Hernández, E., Rodríguez, F. J., y Moral, M. V. (2011). Adaptación escolar de la etnia gitana: relevancia de las variables psicosociales determinantes. Apuntes de Psicología, 29(1), 87-105.

Jones-Schenk, J., y Harper, M. G. (2014). Emotional intelligence: An admission criterion alternative to cumulative grade point averages for prelicensure students. Nurse Education Today, 34, 413-420.

Mayer, J., Salovey, P., y Caruso, D. (2009). Test de Inteligencia Emocional de Mayer, Salovey y Caruso. Madrid: TEA Ediciones.

Oropesa, F., Moreno, C., Pérez, P., y Muñoz-Tinoco, V. (2014). Rutinas de tiempo libre: oportunidad y riesgo en la adolescencia. Cultura y Educación, 26(1), 159-183.

Pulido, F., y Herrera, F. (2015). Miedo e inteligencia emocional en el contexto pluricultural de Ceuta. Anuario de Psicología, 45(2), 249-263.

Siqués, C., y Vila, I. (2014). Estrategias discursivas y aprendizaje de la lengua escolar: un estudio etnográfico en un aula de alta diversidad étnica y lingüística. Cultura y Educación, 26(2), 349-376. 